\title{
Development of the Fast Steering Secondary Mirror Assembly of GMT
}

\author{
Sungho Lee*a, Myung K. Cho ${ }^{\mathrm{b}}$, Chan Park ${ }^{\mathrm{a}}$, Jeong-Yeol Han ${ }^{\mathrm{a}}$, Ueejeong Jeong ${ }^{\mathrm{a}}$, Yang-noh \\ Yoon $^{\mathrm{a}}$, Je Heon Song ${ }^{\mathrm{a}}$, Byeong-Gon Park ${ }^{\mathrm{a}}$, Christoph Dribusch ${ }^{\mathrm{d}}$, Won Hyun Park ${ }^{\mathrm{d}}$, Youra Jun ${ }^{\mathrm{d}}$, \\ Ho-Soon Yang ${ }^{\mathrm{c}}$, Il-Kwon Moon ${ }^{\mathrm{c}}$, Chang Jin Oh ${ }^{\mathrm{d}}$, Ho-Sang Kim ${ }^{\mathrm{e}}$, Kyoung-Don Lee ${ }^{\mathrm{e}}$, Robert \\ Bernier $^{\mathrm{f}}$, Chris Alongi ${ }^{\mathrm{f}}$, Andrew Rakich ${ }^{\mathrm{f}}$, Paul Gardner ${ }^{\mathrm{f}}$, Lee Dettmann ${ }^{\mathrm{f}}$, Wylie Rosenthal ${ }^{\mathrm{f}}$ \\ ${ }^{a}$ Korea Astronomy and Space Science Institute, Daejeon 34055, Republic of Korea; \\ ${ }^{b}$ National Optical Astronomy Observatory, 950 N. Cherry Ave., Tucson, AZ 85719, USA; \\ ${ }^{c}$ Korea Research Institute of Standards and Science, Daejeon 34113, Republic of Korea; \\ ${ }^{\mathrm{d}}$ University of Arizona, Tucson, AZ 85721, USA; \\ ${ }^{\mathrm{e}}$ Institute for Advanced Engineering, Yongin-si, Gyeonggi-do 17180, Republic of Korea; \\ ${ }^{\mathrm{f}}$ GMTO Corporation, 465 N. Halstead Street, Suite 250, Pasadena, CA 91107, USA
}

\begin{abstract}
The Giant Magellan Telescope (GMT) will be featured with two Gregorian secondary mirrors, an adaptive secondary mirror (ASM) and a fast-steering secondary mirror (FSM). The FSM has an effective diameter of $3.2 \mathrm{~m}$ and built as seven $1.1 \mathrm{~m}$ diameter circular segments, which are conjugated $1: 1$ to the seven $8.4 \mathrm{~m}$ segments of the primary. Each FSM segment contains a tip-tilt capability for fine co-alignment of the telescope subapertures and fast guiding to attenuate telescope wind shake and mount control jitter. This tip-tilt capability thus enhances performance of the telescope in the seeing limited observation mode. As the first stage of the FSM development, Phase 0 study was conducted to develop a program plan detailing the design and manufacturing process for the seven FSM segments. The FSM development plan has been matured through an internal review by the GMTO-KASI team in May 2016 and fully assessed by an external review in June 2016. In this paper, we present the technical aspects of the FSM development plan.
\end{abstract}

Keywords: extremely large telescope, Giant Magellan Telescope, Fast Steering Secondary Mirror, optics, optomechanics, mirror support, tip-tilt, image quality

\section{INTRODUCTION}

The M2 Subsystem of the Giant Magellan Telescope (GMT) consists of seven 1.05 meter segments, six off-axis and one on-axis, each forming an imaging pair with its corresponding primary mirror segment to achieve a common system focus. Two types of secondary mirror subsystems are provided: the Fast-steering Secondary Mirror Subsystem (FSMS) and the Adaptive Secondary Mirror Subsystem (ASMS). The FSMS and ASMS assemblies include the FSM/ASM Segments and the M2 Positioner. The FSM and ASM segments share a common optical prescription and a common interface with the M2 Positioning System at the bottom of the M2 Segment Positioners. The M2 Positioning System provides six-degrees-of-freedom motion for the seven segments relative to the telescope top end and its design concept has been revised since the GMT System Level PDR (SL-PDR) in $2014^{[1]}$ as shown in Figure 1. The M2 Positioner is composed of seven short stroke, high precision hexapods called M2 Segment Positioners for segment-to-segment motion and one large stroke, high precision hexapod M2 Global Positioner for positioning the M2 Mirror Cell with all seven segments to compensate for thermal and gravity induced deflections of the Telescope Top End. The FSM and ASM segments attach to the M2 Segment Positioners at a kinematic interface allowing the individual FSM and ASM Segments to be mounted in a repeatable manner.

*leesh@kasi.re.kr; phone: +82 42 865-3354; www.kasi.re.kr

Advances in Optical and Mechanical Technologies for Telescopes and Instrumentation II, edited by Ramón Navarro, James H. Burge, Proc. of SPIE Vol. 9912, 991241

(c) 2016 SPIE · CCC code: 0277-786X/16/\$18 - doi: 10.1117/12.2233710 
The FSM is composed of seven rigid segments each of which is mounted in an individual cell with tip/tilt actuators that provide pointing corrections at frequencies above the control bandwidth of the Telescope Mount ${ }^{[2][3]}$. The main disturbance the FSM mitigates is image motion due to wind disturbances. Individual segment guiding is required to co-align and position the sub-apertures. The FSM tip/tilt actuators provide the precision required for image stacking in the focal plane. The FSMS will be the commissioning secondary mirror system for the GMT and will be installed when the ASMS is off the telescope. Major servicing will be conducted by removing the Telescope Top End together with the M2 Subsystem using the overhead bridge crane.

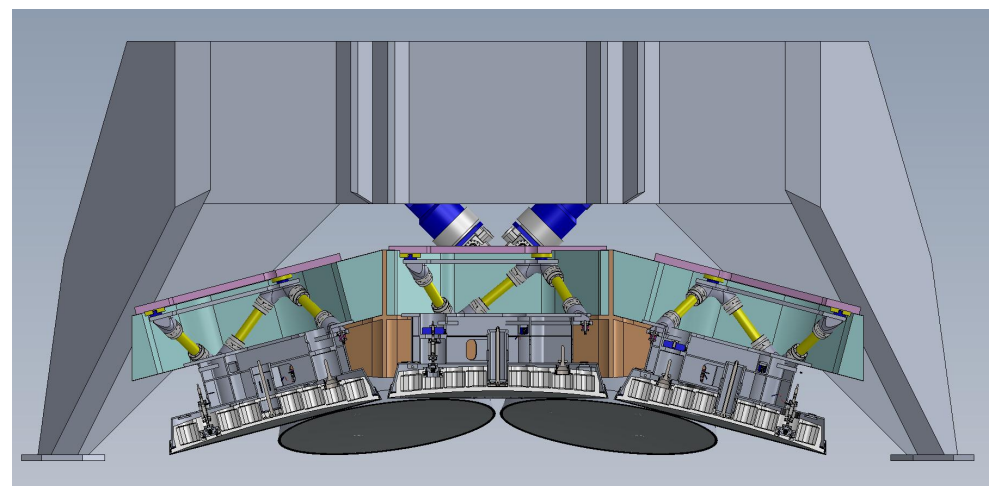

Figure 1. Layout of the GMT M2 Subsystem. The blue hexapod under the telescope top end structure is the M2 Global Positioner and the yellow hexapods in the M2 Mirror Cell are the M2 Segment Positioners to which the FSM Segments are mounted.

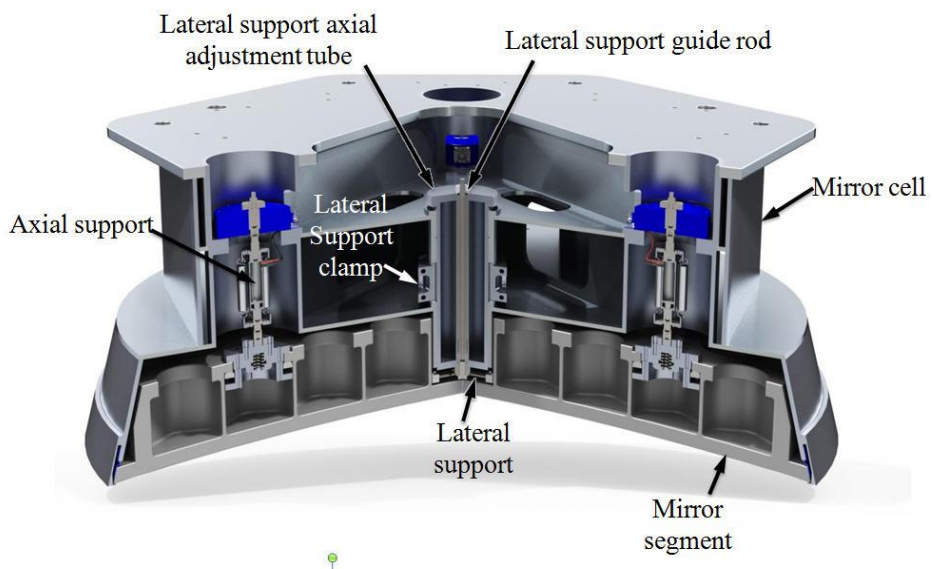

Figure 2. Section view through the design model of FSM at the GMT System Level PDR

During the years from 2009 to 2014, the Korea Astronomy and Space Science Institute (KASI) and its collaborating institutions developed a prototype of one full-size off-axis segment of FSM along with its cell, support system, and a simulator of the GMT top-end to test performance of its tip-tilt control system ${ }^{[4][5][6][7]}$. The goal of this FSM prototype (FSMP) project was to raise technological capabilities in Korea for the essential components in FSM such as a 1-m class off-axis aspherical mirror and a fast tip-tilt control system. While KASI originally aimed to build a prototype based on the GMT design, the completed prototype could not be called literally a real FSM prototype because the final design of the GMT FSM subsystem was not fully matured until the time when KASI needed to freeze the design and start fabrication. However, the experiences of the KASI team with FSMP should be a good starting point for the development of FSM since the design parameters and performance requirements adopted for FSMP were basically identical to those to be adopted for the real system.

\subsection{Organization of the Development Team}

Figure 3 presents the team organization for the FSM development. This setup of participating institutions is basically the same as in the FSMP development, and thus the advantage of utilizing the experiences and facilities accumulated through that previous project can be maximized. In this plan, KASI is leading and managing the FSM project. The National Optical Astronomy Observatory (NOAO), supported by the Korea Research Institute of Standards and 
Science (KRISS) and the Institute for Advanced Engineering (IAE), is collaborating with KASI for the design and development for the cell assembly production. In addition, the University of Arizona (UA) participates in the team to support KRISS with highly advanced and reliable technology for the optical fabrication and metrology.

To optimize the usage of existing resources and capabilities, tasks in some areas may be shared across two or three different institutions. In this case, however, the scope and assignment of commitments and deliverables could become unclear or complicated which is never desirable for a successful project management. A grouping approach, shown in Figure 3, can mitigate this risk and define more clearly the roles and responsibilities of participating institutions in each area as follows.

- Design Group: This group is responsible for design of all components in the FSMS including prototypes, integrated modeling, and design of the associated equipment for the production and handling. The design group is led by NOAO and supported by KRISS for the mirror and its production equipment and by IAE for the cell assembly, the AIT and shipping equipment, and the prototyping components.

- Mirror Production Group: The work scope of this group covers the fabrication and testing of the mirrors, from light-weighting to coating, and its associated equipment. Shipping of the completed mirrors to the AIT site is also the commitment of this group. The mirror blanks will be provided by KASI and all the design models and drawings will be provided by the Design Group. UA will support KRISS for successful fabrication and delivery of FSM segments by providing technical support in fabrication of the FSM mirrors as well as by backing up KRISS for any critical schedule delay.

- Control System Development Group: Design and implementation of the hardware and software system for the FSM control functions is the responsibility of this group. NOAO will take the leading role of this group considering their extensive experiences with a number of similar systems such as the active optics control system for the Large Synoptic Survey Telescope (LSST). Their advanced knowledge on the astronomical systems is also important for close collaboration with the GMT Acquisition, Guide, and Wavefront Sensor Subsystem (AGWS) team and the M2 Positioning Subsystem team to make the FSM working properly as a part of the telescope. The KASI team has performed most relevant and practical tasks with the FSMP. IAE in the KASI team has demonstrated the FSMP control system and they have earned detailed experiences. Thus, for a supporting institution in this group for practical implementation and testing in Korea, IAE could be the firstly considered candidate but the opportunity will also be opened to other institutions with required capabilities of high precision mechatronics.

It is not necessary to define a group for the mechanical production of the cell assembly, AIT equipment, and shipping containers, since all the required activities are part fabrication and assembly, which can be performed through a sole subcontract with IAE.

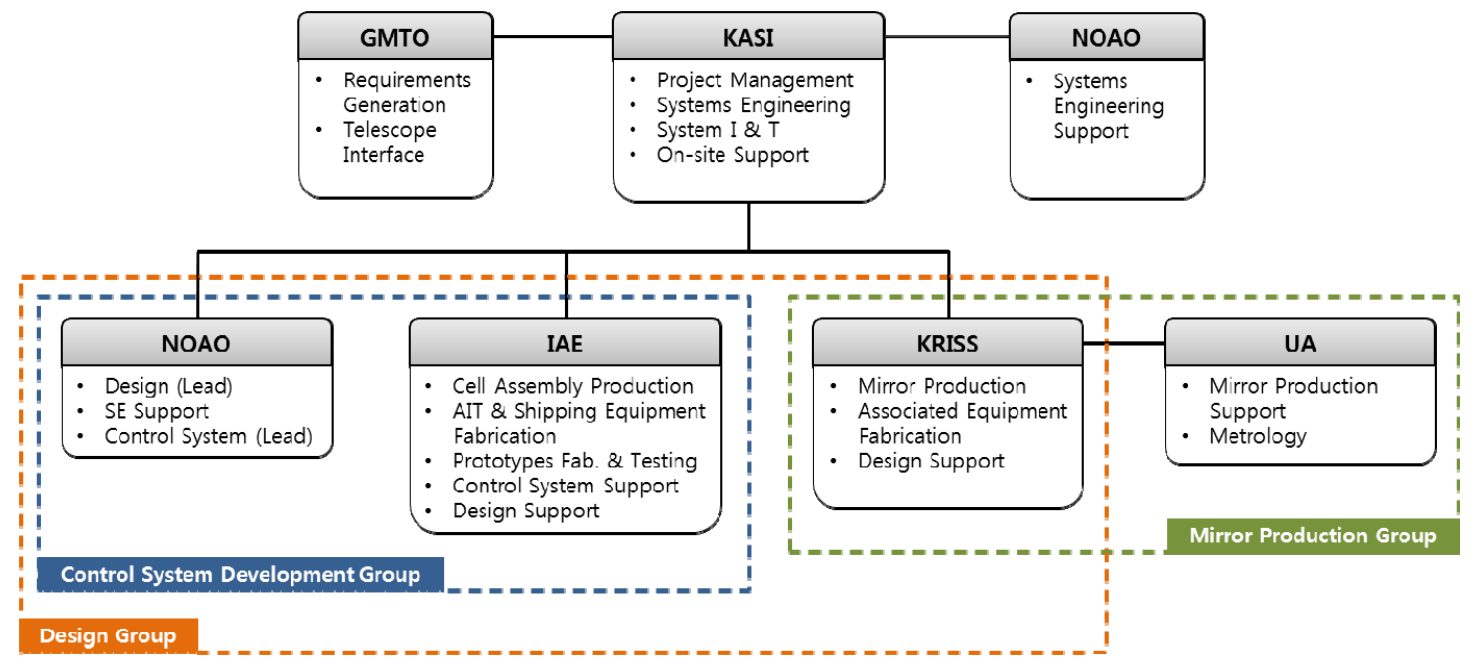

Figure 3. Organization of the FSM development team 


\subsection{Requirements}

Requirements for the design and manufacturing of the FSM are under development by GMTO. A full set of requirements were once drafted by Eric Hansen in $2013^{[8]}$ and, as the design concept of the M2 system has been modified since the GMT SL-PDR ${ }^{[1]}$, the requirements for the FSM are being re-defined accordingly. By now, key requirements have been almost fully developed for optical fabrication and partially completed in other areas as shown in Table 1.

Table 1: Key requirements for FSM

\begin{tabular}{|c|c|c|c|}
\hline Ref \# & Requirement & Value & Tolerance \\
\hline FSM-14755 & Conic Constant & -0.7169273 & Fixed \\
\hline FSM-14756 & Radius of Curvature (RoC) & $4163.901 \mathrm{~mm}$ & $\pm 140 \mu \mathrm{m}$ \\
\hline SLR-15068 & RoC Accuracy & $30 \mu \mathrm{m}$ & $\mathrm{n} / \mathrm{a}$ \\
\hline FSM-15014 & On-Axis CA Diameter & $\geq 1050.3 \mathrm{~mm}$ & Min \\
\hline FSM-14748 & Off-Axis CA Diameter & $\geq 1041.5 \mathrm{~mm}$ & Min \\
\hline FSM-14751 & Decenter Accuracy & $45 \mu \mathrm{m}$ & $\mathrm{n} / \mathrm{a}$ \\
\hline FSM-14764 & Surface Roughness / Micro-Roughness & $<2 \mathrm{~nm}$ RMS & Max \\
\hline FSM-14758 & Mid Spatial Frequency & $<25 \mathrm{~nm} \mathrm{RMS}$ & Max \\
\hline FSM-14759 & High Spatial Frequency & $<8.33 \mathrm{~nm}$ RMS & Max \\
\hline FSM-15024 & Slope Error & $80 \%<0.8 \mu \mathrm{rad}$ & Max \\
\hline FSM-15035 & On-Axis Diameter & $1066.3 \mathrm{~mm}$ & $+/-0.250 \mathrm{~mm}$ \\
\hline FSM-15052 & On-Axis Distance & $0 \mathrm{~mm}$ & $+/-0.225 \mu \mathrm{m}$ \\
\hline FSM-15036 & Off-Axis Diameter & $1057.5 \mathrm{~mm}$ & $+/-0.250 \mathrm{~mm}$ \\
\hline FSM-15051 & Off-Axis Distance & $1087.74 \mathrm{~mm}$ & $+/-0.225 \mu \mathrm{m}$ \\
\hline FSM-14752 & Clocking & $0 \mu \mathrm{rad}$ & $+/-335 \mu \mathrm{rad}$ \\
\hline FSM-14754 & Wedge & $0 \mu \mathrm{rad}$ & $+/-200 \mu \mathrm{rad}$ \\
\hline SLR-14983 & FSM Mass & $250 \mathrm{~kg}$ & $\mathrm{n} / \mathrm{a}$ \\
\hline TS-FSM-4610 & $\begin{array}{c}\text { Tip-Tilt-Piston Range } \\
(0.1 \text { to } 20 \mathrm{~Hz})\end{array}$ & $\geq 12 \operatorname{arcs}$ & \\
\hline TS-FSM-4611 & Tip-Tilt-Piston Resolution & $<20$ mas & \\
\hline
\end{tabular}

Comparison between the old FSMP requirements ${ }^{[5]}$ and the new FSM requirements below leads us to an insight on which point we need an improved (or relaxed) engineering or fabrication performance for the FSM development compared to what we did for the FSMP.

- FSM requirements are defined in much more detail for mirror fabrication and optical testing procedures.

- Current tolerance for radius of curvature (RoC) of $140 \mu \mathrm{m}$ is looser than that for the FSMP $(100 \mu \mathrm{m})$. But, the required measurement accuracy $(30 \mu \mathrm{m})$ is very high and may need a new, improved metrology. 
- Current tolerance for off-axis distance (OAD) of the off-axis mirrors $(225 \mu \mathrm{m})$ is moderately tighter than that for the FSMP $(300 \mu \mathrm{m})$, which may be achievable by the FSMP-applied methodology.

- Current requirement of clear aperture (CA) with respect to the outer diameter of mirror (16 mm margin in diameter which was $8 \mathrm{~mm}$ in the GMT Optical Design in $2013^{[9]}$ ) is significantly relaxed than that for the FSMP (no margin except for $2 \mathrm{~mm}$ in diameter for edge chamfer), which may eliminate concern about an occurrence of edge turn-down during the final figuring process.

- Current requirement of mirror surface error is relaxed than that for the FSMP in that it is now defined as RMS errors in mid- and high-frequency bands while a structure function was required to be met at all frequencies from $1 \mathrm{~mm}$ to $1000 \mathrm{~mm}$ scale in the FSMP development.

- The requirements regarding tip-tilt control are basically the same as those for the FSMP and still under development.

New requirements for the FSM in other areas such as mechanical engineering, vacuum control, software, safety, and environment and detailed ICDs have not been revised yet since the GMT System Level PDR, and will be ready before the next phase of the FSM development starts.

\section{DESIGN PLAN}

The baseline design at the GMT SL-PDR may not be an ideal design for the FSM, but it seems clear that this design is practically the best choice for the project with the limited time and budget considering that all remaining design concerns are not serious or unique but resolvable with existing knowledge and technology. However, it may be also worthwhile to check any possible alternative design concept at this stage of the project, which might be able to provide a better solution without significantly increasing time and cost for its implementation. Therefore, we plan to explore a couple of alternative possibilities before finally fixing the design concept of the FSM at PDR, in parallel with revising and completing the baseline design.

For the baseline design, major revisions and modifications will be made to the mirror, the lateral support and the mirror cell. The optical surface configuration of the mirror will be kept the same; however, design modifications will be in the lightweight pattern, the central hole size, and the flange edge dimension. Among the cell assembly components, designs of seismic restraint, vacuum seal, lateral support and its flexure, and tangential support will mainly be revised. The structure of the mirror cell can be modified significantly to accommodate the changes in the lateral support design and to secure its manufacturability and repeatability of assembly. A newly conceived cell design will be radically simplified and its manufacturability will be highly enhanced by decreasing the number of parts to be fabricated only with simple machining process which can bring down the manufacturing time and costs (see Figure 4). This design reduces blockages or walls that cause inefficient access to inner parts in the previous design at the GMT SL-PDR. During regular maintenance, this design can provide direct access to each single item readily.
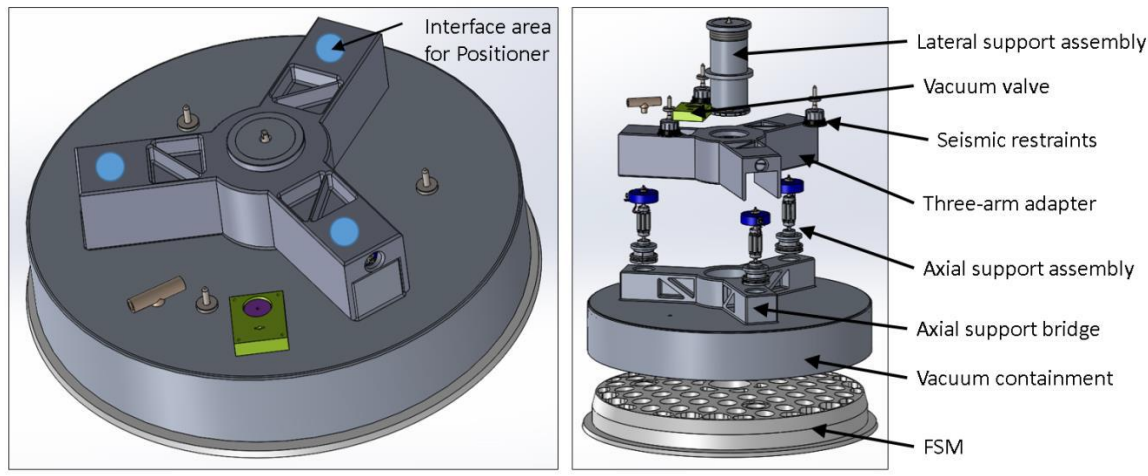

Figure 4. New design concept of the cell assembly for improved manufacturability

The next phases of development, the Delta PDR and Prototyping Phase, begins with analyzing high-level requirements, error budget, and ICDs provided by GMTO to understand them clearly, adjust or supplement if necessary for practical implementation, and derive lower-level engineering specifications and error budget from 
them. A number of trade studies and prototyping studies will be performed during this preliminary design phase to examine the best design concept as well as to make better design choices in the baseline concept and to prove or improve the design of undemonstrated parts in the FSMP project. If a decision is made to change the design concept itself, the FSM development plan including schedule and budget would need to be modified accordingly and the preliminary design phase may need to be extended to develop the newly selected concept into a PDR-level design. More details of the trade studies are described in the following subsections and the prototyping plan in Section 4.

In the detailed design phase after PDR, design models and implementing procedures will be further detailed, optimized, and fixed for the mirror fabrication and the cell assembly. One of the most important tasks in this phase is the integrated modeling and its FEA evaluations to secure the integrity and performance of the FSM as a system working on the telescope. This phase also includes a prototyping study in which a full-scale mirror cell and a dummy mirror will be fabricated and assembled together to confirm and fine-tune the final model of the cell assembly, its fabrication drawings, and the associated procedures before CDR.

The scope of design works for the FSM includes not only the mirror, the cell assembly, and its control system, but also all the associated jigs and equipment such as handling fixtures, fabrication supports, installation stands, tip-tilt testbed frames, shipping containers. The associated hardware will be fabricated and prepared earlier to enable to start the FSM production as soon as the design is ready. Implementation procedures for fabrication, assembly, handling, testing, operation, etc. and detailed plans for associated activities such as acceptance tests, shipping, onsite support, maintenance, etc. will be developed along with the design of hardware and software. This approach is necessary to make the design realistic and avoid any risk of finding problems and contradictions too late in the later stages of the project.

\subsection{Alternative Design Trade Study}

There have been some concerns raised with the vacuum support and its pressure control system in the baseline design about higher cost for continuous maintenance and increasing risks of system malfunction and accident during more frequent services. Considering these complications, a totally different design concept might be able to provide a better and more efficient solution. The following two different concepts will be studied to compare their pros and cons with those of the baseline design. In this trade study, major assessment points would be degree of gravity print through, performance of dynamic tip-tilt operation, specific stiffness, and impacts on the cost and schedule.

- Thick mirror design: If the thickness of a light-weighted glass (or glass-ceramic) mirror blank is large enough with very deep ribs, its mass-to-rigidity ratio becomes so small that the mirror itself may be able to maintain its original shape against the gravity without a vacuum support. The secondary mirror of the 8.2$\mathrm{m}$ Gemini telescopes is an excellent example of this concept, which has been working successfully for more than 16 years since its first light in $1999^{[10][11]}$. The Gemini M2 mirror made of Zerodur, lightweighted with ribs of about $160 \mathrm{~mm}$ deep and $5 \mathrm{~mm}$ thick, was fabricated by REOSC. The high stiffness structure of this mirror was achieved not only by the deep light-weighting but also by the double-arch shape of the backside.

- Distributed force support design: The function of vacuum support is preventing the mirror deformation by holding it with uniformly distributed force at every point which is not supported by the lateral support. There are some examples of implementing the functional merits by using multiple mechanical supports instead of vacuum pressure. Whiffletree type mount is the most typical concept for this kind of distributed force support. Examples for the whiffletree support are segments supports in the $\mathrm{Keck}^{[12]}$ and $\mathrm{TMT}^{[13]}$ primary mirror. For the multiple actuators support concept, the $\mathrm{GMT}^{[14]} \mathrm{M} 1$ and the Magellan ${ }^{[15]} \mathrm{M} 1$ have the similar feature in the active optics system.

\subsection{Baseline Design Trade Study}

In addition to the study for alternative concepts above, trade studies on the following three topics are planned to improve and further develop the baseline design.

- Bonding method: Design choices of material selection, mechanical interface, assembly procedures, etc. can be affected by bonding method. Among the available bonding solutions (RTV, epoxy, and glass-frit bonding), epoxy seems the most preferable choice for the FSM application. NOAO and KRISS are well experienced with the epoxy bonding through a number of previous applications including space missions, and have developed reliable recipes for its treatment procedure. As for the concern with epoxy on its 
ageing or creeping, a literature research would be able to determine the critical timescale of those effects and to assess the consequent risks since it is known that there have been extensive reports of experiments or experiences using epoxy in various fields.

- Reaction force compensation: The degree and characteristics of reaction force by tip-tilt motion will be predicted and assessed carefully along with the revision of the baseline design. In case that a compensation mechanism is determined to be required, a trade study will be performed to select the best solution among a reaction force cancellation system, an active damping system, and a passive damping system. Assessment of cost impact, influence on the FSM and telescope design, and feasibility of implementation will be included in the study. The final decision of adoption would have to be made at the M2 Subsystem level since its demand may be beyond the scale and scope of the FSM segments and also such a mechanism could become a part of the M2 Positioning System.

- Edge sensors: Edge sensors are planned to be applied to the FSM although phasing is not required for the FSM functional purposes, since by this approach edge sensors for the ASM can be verified and prepared well in advance. The KASI team will provide evaluations on the influence of using edge sensors on the FSM mirrors to GMTO for the design consideration of edge sensors. Depending on the locations and shape of edge sensors, the KASI team will need to modify the design of mirror, vacuum seal, and the cell skirt to satisfy the related requirements of alignment and interface.

\section{OPTICAL FABRICATION PLAN}

Overall process flow of optical fabrication for the FSM mirrors is described in Figure 5 along with metrology equipment to be used at each stage.

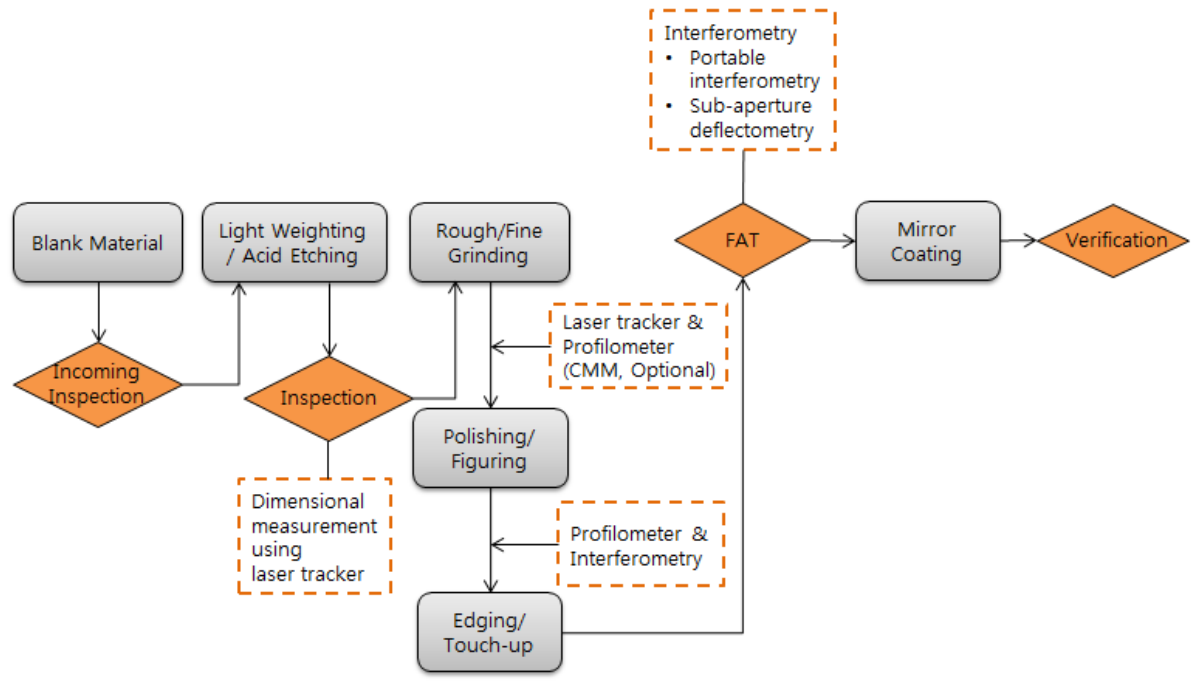

Figure 5. Overall fabrication process of the FSM mirrors

\subsection{Light-Weighting}

Blanks of glass or glass-ceramic will be procured by KASI before the mirror CDR and get prepared for lightweighting after incoming inspection. Light-weighting will be performed using two large CNC generators in parallel. For the first two segments, preliminary light-weighting will start right after the mirror CDR to maximize the schedule efficiency and this process will be finalized according to the finally approved drawing through the CDR responses.

The front surface will be generated to the net surface with a room for optical grinding and polishing. Other surfaces will be etched to ensure the blank strength. To accommodate the edge margin the front surface outer diameter will be oversized by approximately $20 \mathrm{~mm}$ in diameter and will be cut after the processing. KRISS carries numerous experiences in edging down after polishing from many delicate mirror fabrications. The generated surface will be measured using a laser tracker at the center of curvature. 


\subsection{Fabrication Support and Blank Registration}

The fabrication support (or working cell) will be designed to maintain the supporting force to be distributed according to the mirror construction. Also the design will accommodate the allocated error budget. A status monitoring system will be implemented to capture any safety issue.

Particularly each off-axis segment is required to register the orientation referenced to the parent vertex. We are planning to use four SMRs to register the orientation of the mirror and the orientation will be registered to the machine coordinate system as well to be used with the Computer Controlled Polishing (CCP) system.

\subsection{Grinding Phase}

The grinding process consists of two stages, one for rough grinding and the second for fine grinding. The goal of rough grinding is to figure the front surface to the nominal shape of mirror prescription and to remove any defective features on the surface left behind from the curve generating process including sub-surface damage (SSD). The tooling used for all process will be a conventional ceramic tool on flexible plate to accommodate aspherical departure. In the rough grinding stage, a laser tracker will be used to measure the mirror shape at the center of curvature, and a profilometer measurement can guide this process down to a few $\mu \mathrm{m}$ RMS. Figure 6 describes the aspheric departure of the on-axis and off-axis segments of the FSM. As in the figure the center segment has very mild asphericity but the off-axis segment has large asphericity to $1.622 \mathrm{~mm}$ P-V.
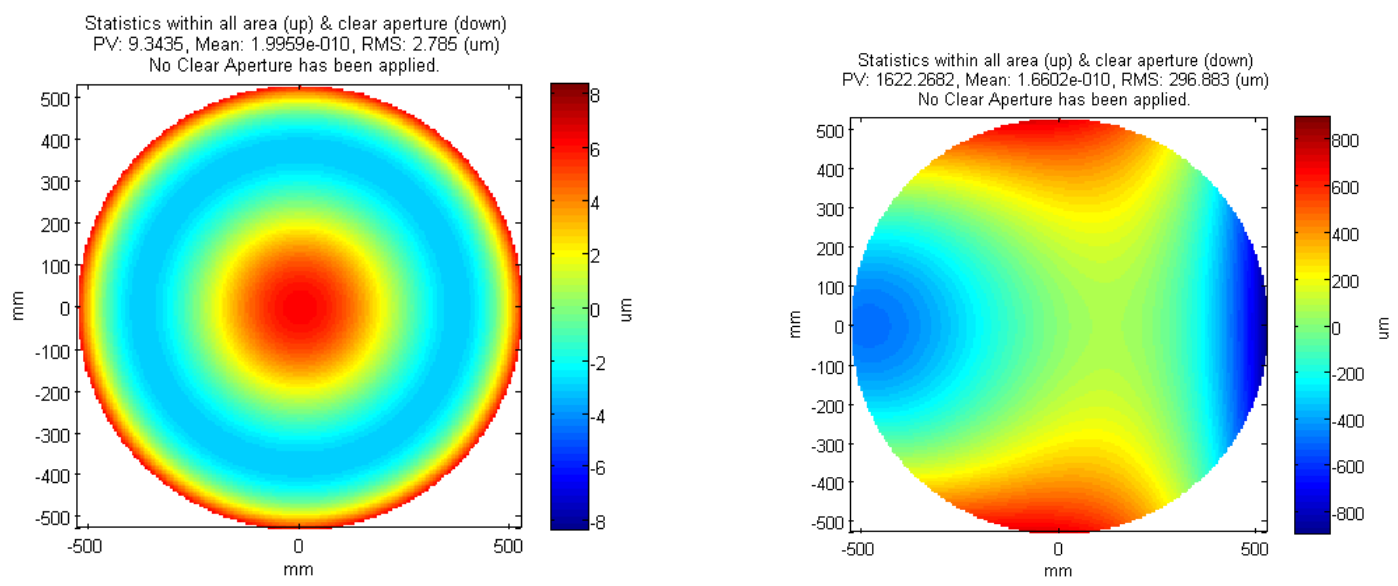

Figure 6. Aspheric departure of the on-axis and off-axis segments of the FSM

The goal of fine grinding is to perform fine figuring and to remove SSD from rough grinding, where the figure accuracy will be less than $1 \mu \mathrm{m}$ RMS and the radius of curvature will be controlled in specification. The KRISS has vast experience in fine grinding, and $45 \mu \mathrm{m}, 25 \mu \mathrm{m}$, and $12 \mu \mathrm{m}$ alumina grits will be used with a conventional ceramic tool on a flexible plate. In the final grinding process, the laser tracker and the profilometer will be used as in the rough grinding process. As grinding phase goes to finer grits, the tool misfit is critical to achieve a smooth surface figure on a steep aspheric surface.

\subsection{Polishing and Figuring Phase}

In the polishing and figuring phase, regular optical pitch and Cerium Oxide will be used for the polishing medium. A CGH test and the profilometer with an interferometric probe will be used to guide the final figuring process. As soon as the surface gets polished out, the profilometer will be featured with an interferometric probe and the CGH test will be performed to provide an initial figure of the mirror surface for the profilometer verification and calibration. Figure 7 presents the profilometer set up for a 1-m class aspherical mirror testing at UA. As shown from recent applications, performance of the profilometer with an interferometric probe is comparable to an interferometry ${ }^{[16]}$. 


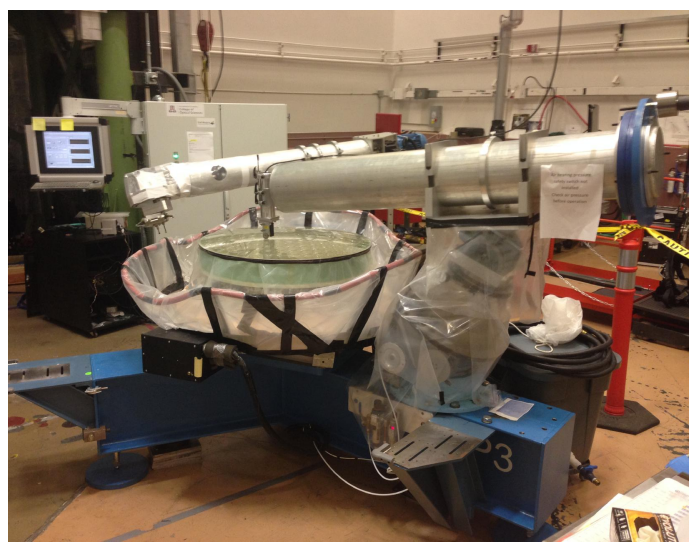

Figure 7. Configuration of the profilometer at UA

\subsection{High Spatial Frequency Figure}

To verify high-spatial-frequency specification, a sub-aperture deflectometry that provides $0.1 \mathrm{~mm}$ spatial resolution will be applied. We are planning to use a similar deflectometry device used for the DKIST primary mirror fabrication ${ }^{[17]}$ shown in Figure 8.

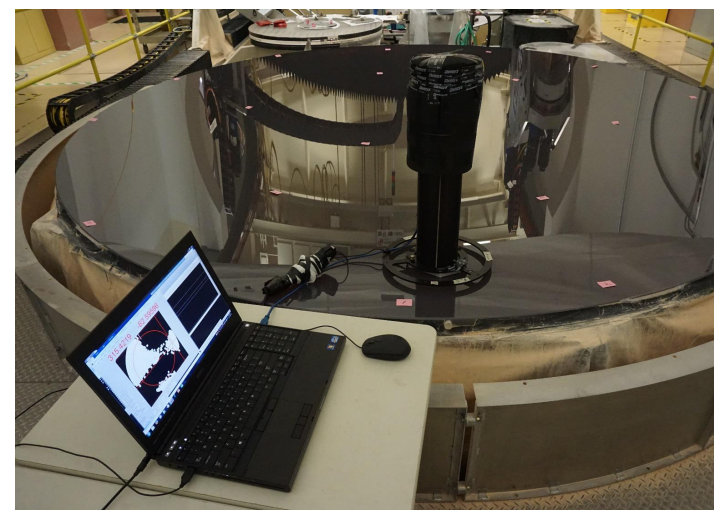

Figure 8. Sub-aperture deflectometry applied to the DKIST primary mirror test at UA

The roughness specification will be tested with a commercial white light interferometry or a portable phase shifting interferometry. Through the FSMP project in the past, the KASI team demonstrated that the mirror was fabricated with fine surface finish using the portable phase shifting interferometer.

\subsection{Fabrication Plan}

The actual fabrication operation will be run in parallel using two CCP systems, and to maximize the processing efficiency multi-shift operation might be applied. The whole time period for the optical fabrication for a segment is roughly presented in Figure 9. In each fabrication process, the appropriate metrologies will be used to cover overall power spectrum requirements as summarized in Table 2. 


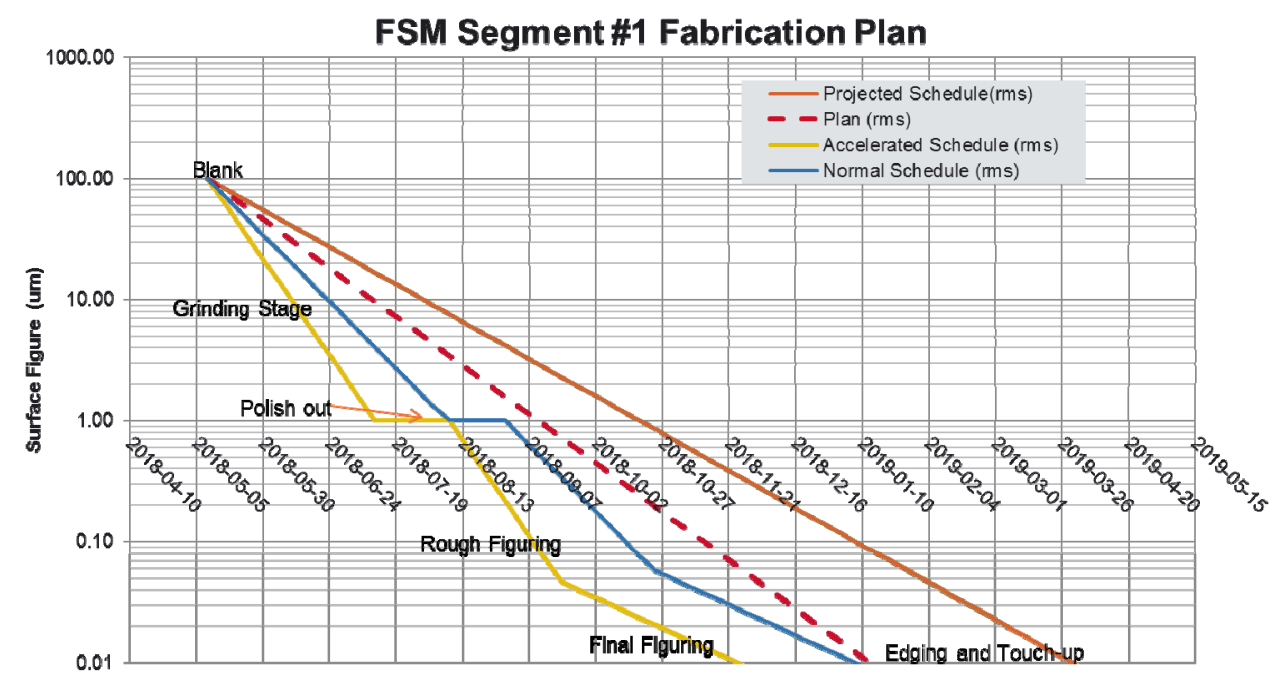

Figure 9. Expected time period for the optical fabrication of a FSM segment

Table 2. Summary of the metrology plan for the FSM mirror fabrication

\begin{tabular}{|c|c|c|}
\hline Fabrication Stage & Primary Metrology & Redundant Metrology \\
\hline Incoming Inspection/Generating & Laser Tracker & CMM (optional) \\
Rough Grinding & Profilometer & Laser Tracker Tracker \\
Fine Grinding & Profilometer & CGH \\
Polishing and Figuring & Profilometer/Interferometric Probe & Profilometer/Interferometric Probe \\
High-Spatial Frequency Figure & Sub-aperture Deflectometry \\
Surface Finish & Portable Interferometry & - \\
\hline
\end{tabular}

\section{MECHANICAL MANUFACTURING PLAN}

In the preliminary design process, the baseline design will be enhanced and optimized, and implementation procedures such as assembly/disassembly procedure will be developed. This design revision should accommodate results from component-level prototyping for seismic stopper, vacuum seal, tangential support and lateral flexure which have not been fully implemented in the FSMP demonstration. KASI has identified certain sub-assemblies and parts which needed further development or revision. A tangential support can be excluded from the prototyping if the anti-clocking performance of the mirror cell indicates a proper torsional rigidity with the new lateral flexure design.

For the break-away clamp system in the axial supports, failure scenarios for a large translational excitation event will be considered and dynamic tests along with FE simulation will be performed for the design validation. This part can be readily tested using the FSMP as a test bench, instead of building a new prototype. Actual breaking away initiation moments and the responses due to external accelerations will be monitored and measured, and recovery performance after an impact will be assessed. Current mechanism design has a balancing feature with sets of springs in push and pull which are preloaded. The set of spring configurations can be parameterized and the breakaway forces vary with the stiffness of the springs. The spring characteristics will be studied and the tests will be done with various spring sets applied.

After the design model becomes matured to meet all the major requirements, the mechanical performance will be verified through a full-scale prototyping of the cell assembly with a dummy mirror. In this process, the repeatability of the cell and mirror assembly/disassembly will be assessed and improved to secure that the performance of the FSM assembly will be maintained acceptable through the multiple assembly and disassembly for the shipping from 
Korea to Italy and to Chile as described in Section 6. The design and drawings will be adjusted and finalized, and the assembly/disassembly procedure can be optimized and fully assessed from the prototyping results. Also, environment tests for the cell assembly will be performed using the full-scale prototype including the tip-tilt performance test. Dynamic environment tests will be conducted with a shake table or by a dynamic simulator for wind buffeting or seismic, and the test results will be compared with the FE integrated model.

Overall process of mechanical part development for the FSM cell assembly is shown as the flow diagram in Figure 10. After the design models and drawings are fully developed, fixed, and confirmed through CDR, the cell assembly fabrication will be processed with the Mandatory Inspection Point (MIP) tests at two levels of the process; at the component level and at the subassembly level. Also the performance of the cell assembly as a system will be measured using the dummy mirror as the Factory Acceptance Test (FAT) at the end of the process. All parts will be fabricated during the production period of the first segment, which includes more schedule contingency to optimize the fabrication and testing procedures and the associated equipment. All manufacturing and machining will be performed with a tight tolerance. Flatness of mounting surface will be specified and position tolerance will be about $25 \mu \mathrm{m}$. A CMM can be used for some critical dimension measurements such as locations of the supports.

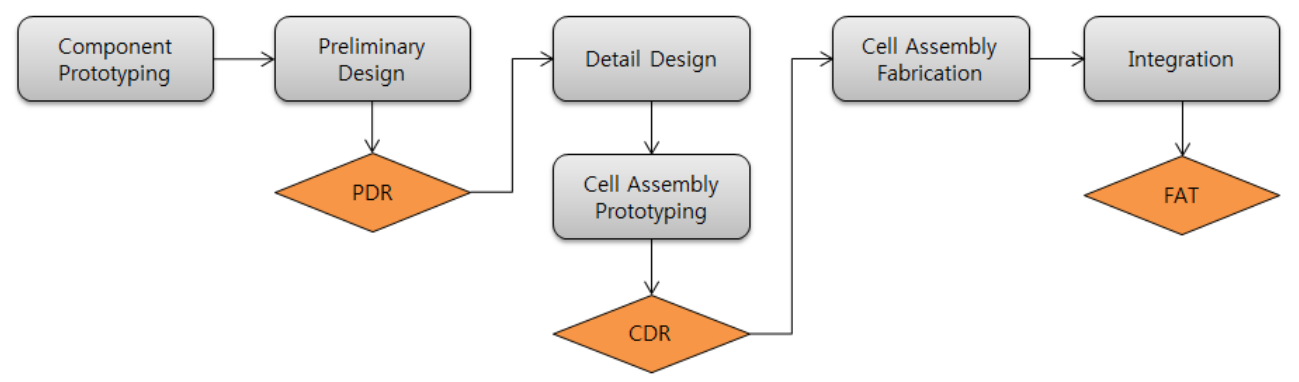

Figure 10. Mechanical manufacturing process for the FSM cell assembly

\section{CONTROL SYSTEM DEVELOPMENT PLAN}

Basic functions of the required control system were well developed through the FSMP project and we plan to perform a further development for the FSM assembly. One of the most important parts among the remaining tasks, other than modifications and optimizations for new requirements, would be an improvement of the testing setup and procedure for the tip-tilt attenuation performance test. A prerequisite for this task is the development of a closedloop control algorithm for real operation on the telescope.

An optimum closed-loop tip-tilt control system should be able to minimize or mitigate any mirror deformation due to the tip-tilt actuation and the effect of tip-tilt reaction momentum. Also the system needs to eliminate any instability associated with mode coupling among the multiple FSM segments as well as meet the main performance requirements. Design of an optimum closed-loop control system requires understanding of the GMT AGWS subsystem, vibrational characteristics of the telescope structure, and detailed dynamic model of the M2 system complex on the top end.

For the tip-tilt control, the FSM control system will interact with the AGWS subsystem in the GMT main control system. The AGWS provides sets of tip tilt error information for the GMT Natural Seeing Fast Guiding Controller. The Fast Guiding controller generates tip-tilt commands and sends them to the FSM control system. The command set contains absolute segment tip-tilt amounts for each FSM segment in the telescope coordinates. Then the FSM system controls actuators according to incoming commands.

Another main function of the FSM control system is vacuum pressure control. The vacuum/pressure control technique for a secondary mirror has been proven by other telescopes such as WIYN ${ }^{[18]}$, Magellan, and DCT ${ }^{[19]}$, and through the FSMP project. We will accommodate their heritage for the FSM control system to implement vacuum/pressure control along the inclination angle and pressure monitoring to feedback the vacuum control. Also temperature sensing at various points on the mirror blank will be included to monitor any temperature variation by heat dissipation or ambient change which may affect the optical performance. Physical connections for components of the FSM control system will be implemented according to the hardware plan in Figure 11. All communications 
between the GMT Device Control Computer (DCC) and IO modules are based on the EtherCAT according to the GMT standard. The connection method between motion controller and EtherCAT interface module is SPI (Serial Peripheral Interface) which is a digital interface bus with very low latency.

We plan to design the FSM control software by the software plan in Figure 11, which consists of a global supervisor module and segment supervisor modules. The global supervisor module receives sets of tip-tilt commands from the GMT Fast Guiding control system which are parsed and distributed to each segment-level supervisor. Also, the global supervisor gathers responses from segment supervisors and sends telemetry messages to the upper level system. The segment level supervisors translate commands into low level commands for hardware. The segment level supervisors also performs the functions of local pressure/vacuum control loop. Interface protocol between the upper level subsystem of the GMT and the FSM system will be defined by the GMTO standard profiles.

Tests for the control system development will be done using the full-scale prototype cell assembly which will be built before CDR. The completed and acceptance tested control system will be ready for integration into the cell assembly system and implementation before the assembly of mechanical components into the first cell assembly.

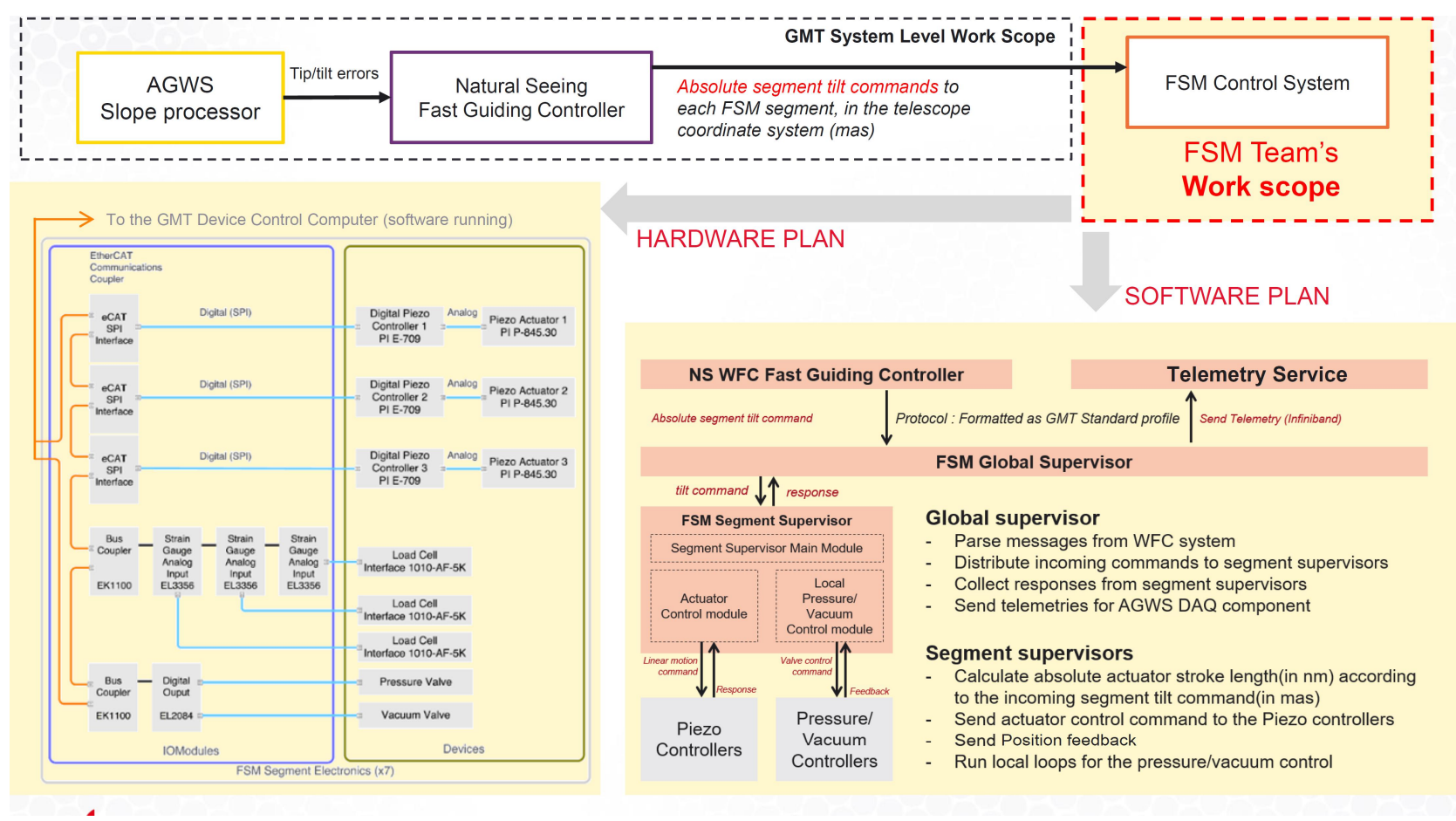

Figure 11. Conceptual development plan of the FSM control system.

\section{SYSTEM AIT AND PRODUCT ASSURANCE PLAN}

Figure 12 shows the workflow from the integration of completed mirror and cell assembly through the final FAT in Korea to the delivery to the M2 Subsystem testing site, and beyond that to the telescope commissioning. In the diagram, the unshaded parts are activities by GMTO to which the FSMS development team will provide technical support.

A mirror, having passed its own FAT at the mirror fabrication site of KRISS, and a cell assembly, tested using a dummy mirror at the mechanical fabrication site of IAE, will be shipped to the FSM AIT site at KASI. The system integration of the mirror and the cell assembly will be performed following the assembly procedure which will be based on the FSMP assembly procedure with some modifications for the new cell design. During the system integration, balancing between three axial supports will be achieved with load cells and alignment of the mirror and cell will be performed by aligning the fiducial makers on the optical surface to the interface surface between the cell and the M2 Positioner using a CMM. 
The factory acceptance test (FAT) for the integrated FSM system will include functional and performance tests of the vacuum control and tip-tilt control with the real glass mirror installed, and optical performance tests using CGH with the optics on the real operational mounts and with the vacuum support applied. All those tests will be performed at two (vertical and horizontal) or more inclination angles to validate the system in operational conditions. The detailed procedure for the FAT will be developed through the design phases including complete and clear definitions of testing conditions and acceptance criteria.

For the AIT and FAT of the FSM, which will be performed in a clean (class 10,000) and temperature controlled (10$30^{\circ} \mathrm{C}$ with accuracy of \pm 0.5 degree) space at KASI, another set of testing equipment such as a CMM, a laser tracker, a CGH, a 4D interferometer, an inclined testbed frame, a testing tower, a vacuum pump and control system is needed other than those for the production processes which should keep working for the later FSM segments in parallel with the AIT activities for the earlier segments. Many of the tools and equipment can be recycled from the FSMP project, but the inclined testbed frame should be re-developed since the current one was designed to be working at a smaller range of inclination angles $\left(0^{\circ}\right.$ to $\left.60^{\circ}\right)$ and is not stiff enough against tip-tilt reaction momentum.

Whenever the FSM needs to be shipped, the mirror and the cell assembly will be disassembled and packaged separately to protect the mirror securely from bumping into the surrounding cell structure by any external impact during handling and transportation. By definition, the mirror cannot be perfectly fixed with respect to the mirror cell since the stiffness or safety factor of the lateral support flexure cannot be too much high and the axial support flexures should allow free lateral motion for their original function of tip-tilt motion.

As shown in Figure 12, this means that assembly of the mirror and the mirror cell will be performed at least three times from the initial AIT to the telescope commissioning. Therefore, repeatability of the assembly/disassembly procedure is very important to the FSMS and all the alignment and performance achievements should be easily reproduced through that procedure without any time-consuming effort or special skill. In this consideration, the fullscale prototyping is planned before CDR to fulfil this requirement in the design and procedures, and the whole integration and testing procedure of AIT at KASI will be cycled twice to check the repeatability.

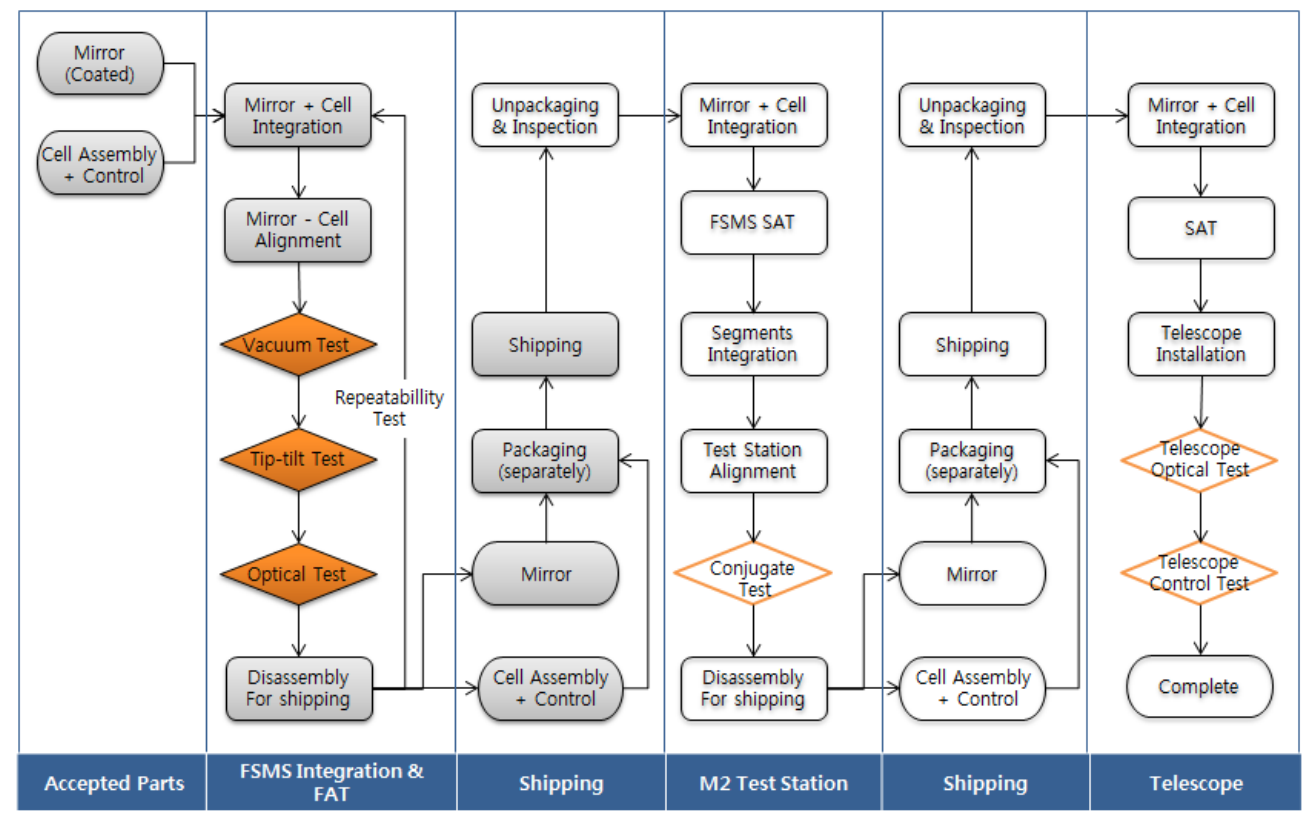

Figure 12. System AIT and commissioning process for the FSM. Unshaded parts after the first shipping are the GMTO activities for the M2 Subsystem integration and telescope commissioning which will be supported technically by the KASI team. 
Thorough Product Assurance (PA) management is essential for the successful delivery of the FSM segments; to meet the requirements of each segment as well as to keep the uniform quality throughout the seven segments which is important to secure the quality of stacked images on the focal plane. As shown in Table 3, our PA plan includes considerations for the safety of human and other resources and the reliability of the system over a long timescale of the GMT lifetime. To operate the PA plan, the PA manager will work with the part manager in each area (mirror production, cell assembly production, control system, and AIT) and collaborate with the Quality Assurance (QA) team of each subcontracted institution.

Table 3. Scope of the PA management for the FSM

\begin{tabular}{|c|c|c|c|}
\hline \multirow{2}{*}{ PA Area } & \multicolumn{3}{|c|}{ Activities } \\
\hline & Design Phase & Manufacturing & Subcontract \\
\hline Part Control & $\begin{array}{l}\text { - Standard/Non-standard parts lists } \\
\text { - Non-standard parts specifications } \\
\text { - Non-standard parts conformance review }\end{array}$ & - Part receiving inspection & $\begin{array}{l}\text { - Requirements flow-down } \\
\text { - Document approval } \\
\text { - Support for issues } \\
\text { - Regular audit }\end{array}$ \\
\hline $\begin{array}{l}\text { Material \& } \\
\text { Process } \\
\text { Control }\end{array}$ & $\begin{array}{l}\text { - Material \& process (procedure) lists } \\
\text { - MUA (Material Usage Agreement) for non- } \\
\text { compliant material } \\
\text { - Procedures document approval }\end{array}$ & $\begin{array}{l}\text { - Material receiving } \\
\text { inspection }\end{array}$ & \\
\hline $\begin{array}{l}\mathrm{H} / \mathrm{W} \& \mathrm{~S} / \mathrm{W} \\
\text { Quality } \\
\text { Control }\end{array}$ & $\begin{array}{l}\text { - Environmental requirements for work areas } \\
\text { (cleanliness, temperature, etc.) } \\
\text { - Workmanship certification (bonding, welding, } \\
\text { soldering, etc.) }\end{array}$ & $\begin{array}{l}\text { - MIP test management } \\
\text { - FAT management }\end{array}$ & \\
\hline Safety Control & $\begin{array}{l}\text { - Identify safety risk factors } \\
\text { - Safety management planning }\end{array}$ & - Operation safety & \\
\hline $\begin{array}{l}\text { Configuration } \\
\text { Management }\end{array}$ & $\begin{array}{l}\text { - Specifications management } \\
\text { - Drawings, parts lists management } \\
\text { - Document control (version \& release) } \\
\text { - Configuration change control (CCB) }\end{array}$ & - As-built parts list & \\
\hline $\begin{array}{l}\text { Reliability } \\
\text { Engineering }\end{array}$ & $\begin{array}{l}\text { - FMEA (Failure Mode \& Effect Analysis) } \\
\text { - Update reliability evaluation at design } \\
\text { reviews }\end{array}$ & $\begin{array}{l}\text { - Failure reporting } \\
\text { - Re-evaluation for } \\
\text { corrective actions }\end{array}$ & \\
\hline
\end{tabular}

\section{CONCLUSION}

As the first stage of the FSM development project, Phase 0 study was conducted from November 2015 to February 2016 to develop of a program plan detailing the design and manufacturing process for seven FSM segments, including WBS, schedule, cost estimation, risk mitigation plan and plans for project management and systems engineering. During the study, experiences and results from the FSMP project were fully reviewed and the lessons learned from it were reflected in the planning for the FSM. The FSM development plan has been matured through an internal review by the GMTO-KASI team in May 2016 and fully assessed by an external review in June 2016. After some further revision of the plan responding to the review recommendations, the next phase of the FSM development, the Delta PDR and Prototyping Phase, is planned to start in August 2016. 


\section{REFERENCES}

[1] GMTO Corp., “GMT System Level Preliminary Design Review,” GMT-SE-RVW-00410 (2014)

[2] Cho, M. K. et al., "Design and development of a fast-steering secondary mirror for the Giant Magellan Telescope," Proc. SPIE 8125, 812505 (2011)

[3] Cho, M. K. et al., "Development of GMT fast steering secondary mirror assembly," Proc. SPIE 9145, 91451M (2014)

[4] Cho, M. K. et al., "Development of a fast steering secondary mirror prototype for the Giant Magellan Telescope," Proc. SPIE 8444, 844420 (2012)

[5] Kim, Y. -S. et al., "Development status of the prototype of the GMT fast steering mirror," Proc. SPIE 8415, $84150 \mathrm{~B}(2012)$

[6] Corredor, A. et al., "Optomechanical analysis and testing of a fast steering secondary mirror prototype for the Giant Magellan Telescope," Proc. SPIE 8836, 88360U (2013)

[7] Kim, J. et al., "Development of a FSMP mirror assembly," Proc. SPIE 9582, 95820K (2015)

[8] GMTO Corp., "Fast Steering Mirror Requirements," GMT-TEL-REQ-00508 (2014)

[9] GMTO Corp., "GMT Optical Design," GMT-SE-DOC-00010 (2013)

[10] Otto, W., "Testing of the Gemini secondary mirrors," Proc. SPIE 3739, 325 (1999)

[11]Carter, C. J. et al., "The Gemini secondary mirror tip/tilt system: past, present, and future," Proc. SPIE 7019, $701921(2008)$

[12] Mast, T. S. and Nelson, J. E., "Fabrication Of The Keck Ten Meter Telescope Primary Mirror," Proc SPIE 0542, 48 (1985)

[13] Williams, E. C. et al., "Advancement of the segment support system for the Thirty Meter Telescope primary mirror," Proc. SPIE 7018, 701810 (2008)

[14]Hull, C., "GMT primary mirror support," Proc. SPIE 9145, 91451H (2014)

[15] Martin, H. M. et al., "Primary mirror system for the first Magellan telescope," Proc. SPIE 4003, 2 (2000)

[16] Oh, C. J. et al., "Fabrication and testing of the $4.2 \mathrm{~m}$ off-axis primary mirror of DKIST," Proc. SPIE, this volume (2016)

[17] Huang, R. et al., "Deflectometry measurement of Daniel K. Inouye Solar Telescope primary mirror," Proc. SPIE 9575, $957515(2015)$

[18] Roddier, N. A., "WIYN telescope active optics system," Proc. SPIE 2479, 364 (1995)

[19] Smith, B. et al., "The active optics system for the Discovery Channel Telescope," Proc. SPIE 7739, $77391 \mathrm{~T}$ (2010) 